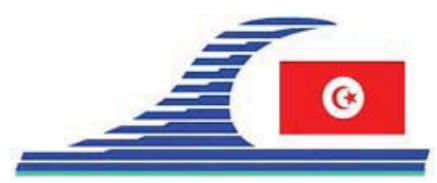

Conférence Méditerranéenne Côtière et Maritime EDITION 1, HAMMAMET, TUNISIE (2009)

Coastal and Maritime Mediterranean Conference

Disponible en ligne - http://www.paralia.fr-Available online

\title{
Atlas hydrodynamique du littoral Languedoc-Roussillon
}

\section{Cyril VANROYE ${ }^{1}$, Frédéric BOUCHETTE ${ }^{2}$}

1. Direction Régionale de 1'Equipement du Languedoc-Roussillon, Service des Espaces Littoraux, Unité Aménagements et Risques Littoraux, 520, allée Henri II de Montmorency, 34064 Montpellier Cedex 2, France. cyril.vanroye@developpement-durable.gouv.fr

2. Université Montpellier II, GLADYS Géosciences,UMR CNRS-UM2 5243, 60 place E. Bataillon, 34095 Montpellier Cedex 5, France.

bouchette@gladys-littoral.org ;www.gladys-littoral.org

\section{Résumé :}

Pour bien appréhender les phénomènes d'érosion et améliorer la gestion des risques, il est indispensable de connaître les climats de vagues et leur impact sur le rivage. La mise en place de réseaux pérennes de mesure de vagues permet de préciser les états de mer et de fournir les historiques de données. Les conditions de houle et de courant à la côte et leur traduction en matière de risque et de transport sédimentaire ne peuvent être observées de manière continue dans le temps et exhaustive dans l'espace. Une méthode possible consiste à fixer des conditions de forçages caractéristiques sur une zone donnée et à recourir à la modélisation pour en préciser les caractéristiques.

C'est l'objectif poursuivi par l'Atlas Hydrodynamique du Golfe du Lion développé dans le cadre du projet européen BEACHMED-e (2009). Plus précisément, cet Atlas rasssemble :

- une cartographie numérique de l'hydrodynamique pré-littorale et littorale forcée par les régimes hydro-météorologiques les plus fréquents

- une présentation détaillée des processus hydro-météorologiques généraux ainsi que des zones retenues

- une présentation des outils, des instruments, des campagnes de mesures réalisées et une revue critique des résultats obtenus

Il synthétise l'ensemble des informations (vagues, courant de circulation et surcote) utiles aux gestionnaires et permettant d'améliorer la compréhension des phénomènes hydrodynamiques. L'élaboration de cet Atlas repose sur l'articulation de campagnes de mesures long terme et court terme ainsi que sur la combinaison d'outils numériques adaptés à chaque échelle de modélisation.

Il constitue à ce titre un résultat original qui met à la portée de tous et de manière opérationnelle des travaux d'étude et de caractérisation hydrodynamique poussés.

\section{Mots-clés :}

Dynamique côtière - Modélisation numérique

$$
\text { DOI: } 10.5150 / \mathrm{cmcm} .2009 .041-1
$$




\section{Qu'est-ce que l'Atlas Hydrodynamique du littoral Languedoc-Roussillon?}

On désigne par Atlas Hydrodynamique du littoral un ensemble d'informations présentées sous la forme de documents (textes explicatifs, diagrammes synthétiques et cartes de grandeurs physiques) utiles et pertinentes pour la compréhension de l'hydrodynamique en zone littorale. Plus précisément, un Atlas Hydrodynamique est aussi une base de données web permettant un accès à l'ensemble de ces informations au format numérique.

La fonction principale d'un Atlas Hydrodynamique du littoral est de permettre à tout gestionnaire du littoral de caractériser les effets du vent et des vagues à l'approche de la côte, sur l'ensemble du littoral.

Par ailleurs, l'idée est de réaliser un outil adapté aux besoins des gestionnaires et de permettre ainsi :

d'avoir une vision claire et rapide de l'ensemble des processus hydrodynamiques à différentes échelles spatiales;

de garantir l'assurance que les informations produites reposent sur des données fiables, et non pas sur des données approximatives résultant d'études anciennes, peu ou pas contraintes;

de connaître exactement la fiabilité des documents constituant l'Atlas, sans complaisance pour les outils utilisés et les résultats obtenus;

d'obtenir des informations plus précises sur des zones littorales essentielles.

Les zones portées dans l'Atlas doivent être représentatives des différents types de segments littoraux sur lesquels les gestionnaires travaillent : littoral en érosion, littoral soumis à l'envahissement de la terre par la mer, littoral en accrétion, littoral aménagé ou en cours d'aménagement, littoral naturel soumis à de fortes contraintes environnementales.

Le travail de conception d'un tel Atlas Hydrodynamique s'organise autour :

de la réalisation de campagnes de mesures hydrodynamiques en zone littorale et sur le proche plateau interne en Languedoc-Roussillon;

de la mise en oeuvre de plusieurs outils de modélisation hydrodynamique à ces différentes échelles spatiales.

Les campagnes de mesure servent d'une part à valider les résultats issus de la modélisation, et d'autre part à déterminer quels sont les états de mer et les conditions de circulation les plus caractéristiques sur les différentes zones considérées. Dans l'Atlas, on parle de conditions académiques pour désigner ces climats de vagues et ces conditions de vent qui ne sont pas nécessairement mesurées à un moment donné sur le littoral, mais sont représentatives des tendances hydrodynamiques sur une portion de littoral.

Les simulations numériques permettent de caractériser l'hydrodynamique à l'échelle des différentes zones étudiées et de proposer des résultats pour des conditions académiques pour lesquelles on ne dispose pas de données mesurées. 


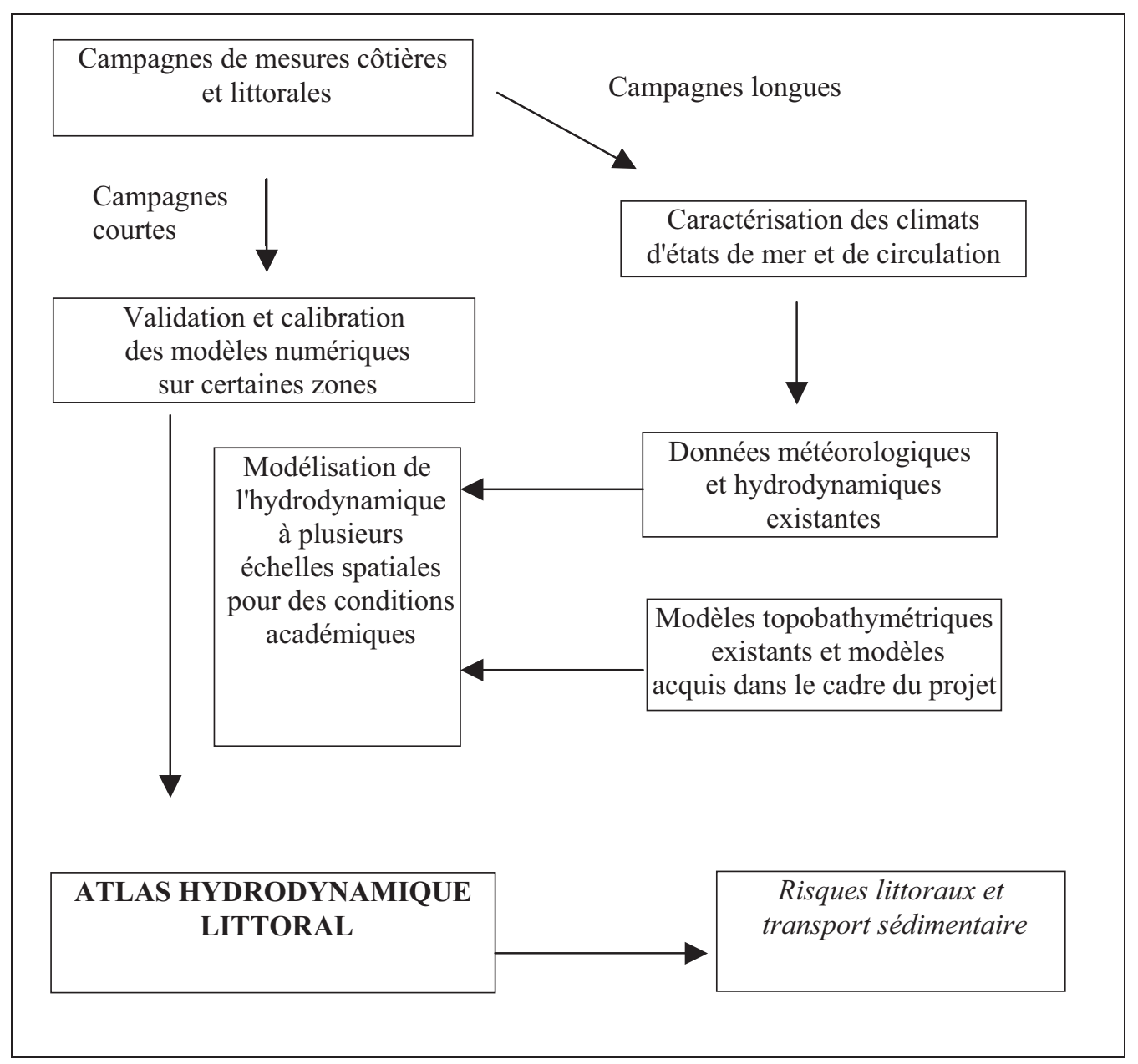

Figure 1. Le principe de réalisation de l'Atlas Hydrodynamique du littoral du Languedoc-Roussillon.

\section{Contenu de l'Atlas Hydrodynamique}

Un travail de fond sur les modèles numériques retenus initialement pour la réalisation de l'Atlas Hydrodynamique a permis d'arrêter une liste de grandeurs physiques qui peuvent être correctement définies par la modélisation en zone de plateau interne et de littoral (hors zone de surf) :

les caractéristiques des vagues ;

les caractéristiques de la circulation 2D;

les caractéristiques de la circulation 3D.

Ces grandeurs physiques sont présentées sous forme de cartes et de coupes associées à un commentaire succinct pour les différentes zones étudiées. Le commentaire permet de porter un regard critique sur les résultats numériques et montre les caractéristiques essentielles de l'hydrodynamique.

Ces documents sont le cœur de l'Atlas Hydrodynamique qui constitue, ce faisant, une cartographie numérique de l'hydrodynamique sur le proche plateau continental et la 
zone littorale, forcée par les régimes hydro-météorologiques typiques du LanguedocRoussillon.

L'Atlas contient des informations complémentaires qui permettent de connaitre la pertinence et les limites des simulations réalisées afin de garder un regard critique lors de l'utilisation de ces données cartographiques. Il comprend en particulier des exemples de validations des modèles et une présentation de la physique sous-jacente aux modèles utilisés.

L'Atlas Hydrodynamique repose sur l'utilisation d'un modèle de circulation 3D appelé Symphonie couplé à un modèle de propagation de vague RefDif modifié pour la réalisation de l'Atlas. Le couplage entre la circulation et a été réalisé selon l'approche de Mellor dans le cadre des travaux de DENAMIEL (2006). Ces modèles simulent une circulation 3D, et une houle monochromatique. Ils ne prennent pas en compte la génération des vagues par le vent local.

L'Atlas Hydrodynamique n'est pas un outil qui permet de reproduire exactement un événement météorologique et ses conséquences exactes en terme d'hydrodynamique. Toutefois, il propose une validation des modèles numériques sur des exemples réalistes comme une succession de tempêtes en décembre 1999, ou celle du 3-4 janvier 2008. Les résultats obtenus montrent que l'outil est bien adapté à l'objectif fixé, notamment au niveau du couplage entre les effets du vent et les effets des vagues.

L'Atlas hydrodynamique repose sur des simulations à différentes échelles, emboîtées les unes dans les autres. Pour chaque forçage académique considéré, des simulations ont été réalisées à 3 échelles globales (Golfe du Lion et plateau externe). Mais seules les échelles plus locales sont exploitées sous forme de cartes (plateau interne et littoral). Cette technique d'emboîtement permet d'une part de prendre en compte les effets à l'échelle du Golfe du Lion (comme l'effet des fleuves sur la circulation) jusque dans les simulations très littorales, et d'autre part d'obtenir un raffinement de la résolution à la côte.

\section{Références bibliographiques}

DENAMIEL C. (2006). Modélisation hydrodynamique $3 D$ en zone pré-littorale: Caractérisation des effets des houles de tempête sur la circulation océanique. Thèse de l'Université Montpellier 2, 424 p.

BEACHMED-e (2009). Rapports du sous-projet NAUSICAA [URL http://www.beachmed.it ] 